\title{
Maximum Distance Band Selection of Hyperspectral Images
}

\author{
Shahram Latifi \\ ECE Dept, UNLV \\ 4505 Maryland Parkway \\ Las Vegas, NV 89154
}

\author{
Steven Wilson \\ ECE Dept, UNLV \\ 4505 Maryland Parkway \\ Las Vegas, NV 89154
}

\begin{abstract}
Hyperspectral Imaging has been advanced by recent improvements in airborne imaging hardware. Early airborne HSI datasets such as Indian Pines, have a relatively low spatial and spectral resolution and are useful primarily for research purposes. Higher resolution and lower sensor noise has become the industry standard. Since there is more high quality data available, less emphasis can be placed on denoising and pixel unmixing, and the problem becomes one of computational complexity. Therefore, there is a need for preprocessing methods which reduce the amount of raw data processed by target detection algorithms. The purpose of this research is to propose a method of maximum distance automated band selection in order to preprocess hyperspectral image cube data, and present the results when compared to those using the entire data set. The goal is to significantly increase the accuracy of target detection using a Robust Matched Filter (RMF) while at the same time reducing the computational time required to process the data.
\end{abstract}

\section{General Terms}

Image Processing, Target Detection

\section{Keywords}

hyperspectral, imaging, automated, standoff, target, detection,

\section{INTRODUCTION}

The research purposes are to establish a robust method for standoff target detection using airborne Hyperspectral Imaging (HSI). There has been a lot of effort placed into target detection algorithms, and denoising of data, but the scope of this paper is from a data processing point of view. A large hyperspectral image containing hundreds of thousands of pixels, each having hundreds of spectral bands is to be processed using a target detection algorithm. If one compares each pixel's spectra against all of the library components, this can be quite time consuming. In the Avon dataset, there are nearly 320 by 5200 pixels. Using Standard Matched Filter (SMF) [5] target detection, a 80 pixel by 40 pixel area takes about 33 seconds to classify. Extending this same rate of detection to the entire scene, it would take nearly 4 hours to classify this entire image. This is unacceptable for real-time and security applications. A band selection method is proposed to decrease the processing time required for successful target detection of a large airborne data collect.

\subsection{Materials spectra library}

Many target detection algorithms rely on a training method that must be completed to begin classifying the materials in a scene, which relies on a priori information. A small subset of the data is used in order to classify the remaining pixels. This requires the band selection process is driven by the types of data in the scene, and must be done manually. A common use for this method are land-use classification [6].

Instead a library of lab collected materials spectra is compiled, so there is no reliance on manual training data to guide proposed target detection. This is imperative, because there may be several targets of interest, there i no prior information since the types of materials may be varied. For security applications, there is also a limited time in which the target detection can be performed, so taking multiple passes through the image is out of the question. Therefore, the algorithm must be guided by the informational quality of the bands which are selected in order to classify the majority of targets in the scene in general.

\subsection{Airborne Imaging Dataset}

The most common collection method is the Airborne Visible / Infrared Imaging Spectrometer or AVIRIS. Images collected through AVIRIS, such as the Indian Pines dataset [1] contain 224 wavelength bands between $400 \mathrm{~nm}$ to $2500 \mathrm{~nm}$ [2]. Other data collection hardware such as the ProSpecTIR [3] provide up to 360 wavelength bands in the same range. The Avon, New York dataset [4], provided by the Rochester Institute of Technology (RIT), utilized this type of airborne data collect to improve spectral resolution over previous publicly available datasets.

\section{METHOD}

An automated band selection (ABS) method is presented to reduce the amount of bands significantly from the original raw image data. Authors are guided by previous works which rely on data quality [7], and specifically, the use of eigenvalues of a band in the HSI datacube [8] to get the most out of the data. The process begins by removing bands which have a low amount of data content, based on the eigenvalues of each spectral layer. This will complete the task of removing the range of bands which are corrupted by noise due to atmospheric scattering, as well as bands which have low spatial correlation. Once the noise bands are removed, the remaining bands will be further limited by maximum distance criteria. What is expected to be achieved by doing this is to reduce the complexity of calculations for target detection using SMF. The same amount of calculations will be performed, but they become less complex because each comparison against the library of materials spectra will contain less reflectance values.

\subsection{Metrics for Comparison of Band Selection Methods}

In order to compare the target detection results from the band reduced images, and the original image, metrics for comparison needs to be defined. First, the target overall accuracy [9] (OA) is defined as the percent of pixels which 
are correctly classified from the scene, when compared to the total amount of pixels of that class defined in the ground truth. Similarly, the average accuracy [16] (AA) is used to emphasize the amount of true negative pixels in the scene, which may become a bit of an issue for the dataset due to high levels of noise. It is for this reason that a higher emphasis will be placed on the OA metric, but will include the equations for both below.

$$
\begin{aligned}
& O A=\frac{\text { True Positive }+ \text { True Negative }}{\text { True Positive }+ \text { False Negative }+ \text { True Negative }+ \text { False Positive }} \\
& A A=\frac{0.5 * \text { True Positive }}{\text { True Positive }+ \text { False Negative }}+\frac{0.5 * \text { True Negative }}{\text { True Negative }+ \text { False Positive }}
\end{aligned}
$$

Equation 1: Calculating the overall and average accuracy of target detection results.

Next, the computation time for the automated band selection and target detection algorithm are summed to give the runtime definition. So it is important that both the band selection method is completed quickly, but also that it is completed in a way that reduces target detection runtime. This band selection would be done on an image by image basis, so there is no way to predict which bands will be selected a priori. The amount of computation time will be compared to the amount of time in which the target detection algorithm takes to analyze the original dataset with no preprocessing.

\subsection{Creating a Ground Truth}

A ground truth result must be obtained from the original RIT dataset, which will act as the template for which the target detection results are compared. Such a task would be difficult given that the ground truth library of materials spectra provided contains over 170 samples. the library is reduced greatly to 12 samples to make it possible for manual classification. A few of the samples in the library are not necessarily present in the scene, and are chosen due to their similarity to other materials present in the scene. For example, the red tarp is not present in this area of the dataset; however, it is very spectrally similar to the white tarp, and often the white tarp is misclassified as the red tarp. This is shown in figure 1.

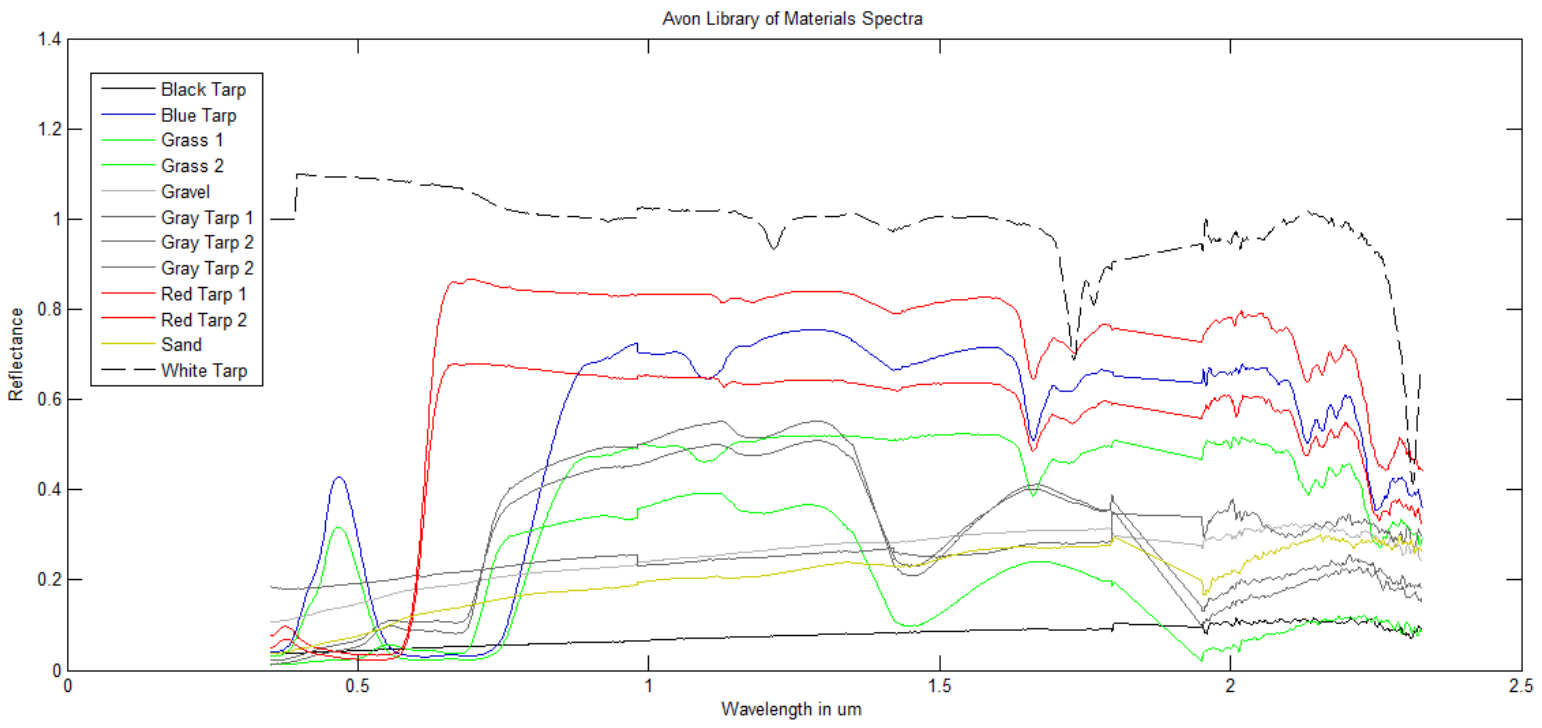

Figure 1: The 12 spectral library bands collected from Avon ground truth data.

\subsection{Theory of Band Selection}

By removing bands in the scene from the calculations, the assumption is made that the majority of the wavelength bands contain for the most part redundant data. Since it has been suggested that only 15 wavelength bands are required to represent HSI data [10], this means that the majority of bands in the image provide less relevant information for target detection. This means that to maintain satisfactory target detection results, only about $5 \%$ of the 360 total original bands contained in the RIT Avon dataset is needed. This value of 15 bands is used as a guide to begin the ABS process, but its validity is evaluated in the results section.

\subsection{Eigenvalue Band Reduction}

It is observed that there are two distinct parts to the band selection process. The first step is band removal, in which some wavelength bands with the lowest first eigenvalues is chosen for removal, meaning that they have a low spatial correlation. Generally, the eigenvalues of bands taken from a natural scene with low noise will be relatively high. due to heterogeneous areas of background which cause large groups of surrounding pixels to have similar values. Therefore, it is known that any low eigenvalues that occur are instead caused by noise in the image. If the bands were to be examined in the range of $1.35-1.42 \mathrm{um}$, and $1.8-1.95 \mathrm{um}$, it is found that they have the lowest eigenvalues. These are the bands that are removed during preprocessing since they are corrupted high levels of noise caused by atmospheric scattering [11].

Next, selection of a threshold value lambda is discussed. Since eigenvalues are raw measure of correlation between values in a matrix, the result is not limited in magnitude. It is decided to represent all eigenvalues and lambda as a ratio with maximum value 1 with respect to the largest eigenvalue evaluated in all 360 bands. The selection of this lambda value 
is limited by a minimum value of zero, which clearly does not eliminate any bands from the removal process, and a maximum which is determined by how many bands are available after this band reduction process. If the final number of bands required is 25 , then the ratio lambda value must be small enough that the band reduction process allows for this amount. This has been determined to be about 0.4 for the RIT subset that are used. As the threshold, lambda, for which bands are selected is increased, more bands are eliminated. These additional bands that are removed generally have low spatial correlation due to high levels of noise, making them very difficult to characterize. Finally, when this process is complete, there is a group of high quality bands that is substantially smaller than the original group of 360 bands.

\subsection{Maximum Distance Band Selection}

In the second step of the ABS process, bands that are most dissimilar and contain the little redundant information are selected. It is necessary to choose the bands not only based on their quality, but also their ability to represent a maximum number of materials in the scene. For example, in a scene that has large amounts of grass, the green bands from 500nm $570 \mathrm{~nm}$ may all have the highest eigenvalues. This is done by finding the bands that have the maximum distance between their reflectance and wavelength values. Similar to a clustering algorithm, the group of selected bands forms the selection process for the next bands, except that when a new band enters the "cluster", it aids the algorithm in finding the farthest possible match from those bands already selected. This is a relatively simple selection process which the reflectance and wavelength values of each pixel are treated as orthogonal, and with equal unit magnitude. Then the distance between bands is sum of the square roots of the distance between their wavelength and reflectance values. Each selected band is summed to the average of the cluster, and the new band which is to be evaluated will compare to this singular pair of values. This is shown in equation 1. Using this method, there are no bands which are duplicated during the selection process.

$$
\text { Distance }=\operatorname{Norm}\left(\left(r_{\text {cluster }}\right)-\left(\frac{1}{\max \left(r_{1}\right)}\left(r_{1}\right)\right)\right)^{2}+\left(\left(\lambda_{\text {cluster }}\right)-\left(\frac{1}{2.50}\left(\lambda_{1}\right)\right)\right)^{2}
$$

Equation 1: Calculating the distance between new bands entering the maximum distance cluster.

Since the values that have already been added to the cluster are with respect to a unit magnitude, the weight must only be applied to the incoming band values. The only exception is the first band in the cluster, which must be weighted itself before being compared to other incoming bands. The weighting value $1 / 2.50$ is based on the maximum wavelength of 2.50um for the SpecTIR collection hardware used in the RIT dataset.

\section{RESULTS}

itmust discuss two factors which determine the effectiveness of this algorithm: The OA and AA of target detection, and the computational time comparisons that follow. First, it is established that the band reduction is either beneficial or causes no change to the accuracy of target detection results, while decreasing the computational time by a substantial amount. In figure 2, a satellite image obtained from ARCGis Flexview [12] displays the flight paths over the AVON data collect site used in the RIT Share project. Since there is no pixel by pixel outline of the ground truth data, it had to be recreated based on the satellite imagery and visual comparison. The manually assigned ground truth image for this scene is shown in figure 3 , and appears approximately 45 degrees counter-clockwise with respect to the satellite image.

There are three detection results to compare: first is the control group, in which target detection is performed on the entire subscene. The results are compared against the ground truth data in figure 3 . The next results come from the automated band selection method which has two stages: band reduction based on quality threshold lambda, and band selection based on the maximum distance algorithm and total number of output bands. One needs to study the effects of varying the value lambda used in the band reduction process, then follow by comparing the effect of varying the number of bands used in the band selection process. Each target detection is performed using the SMF with 12 spectral library materials.



Figure 2: Satellite image of the AVON airborne imaging site. 


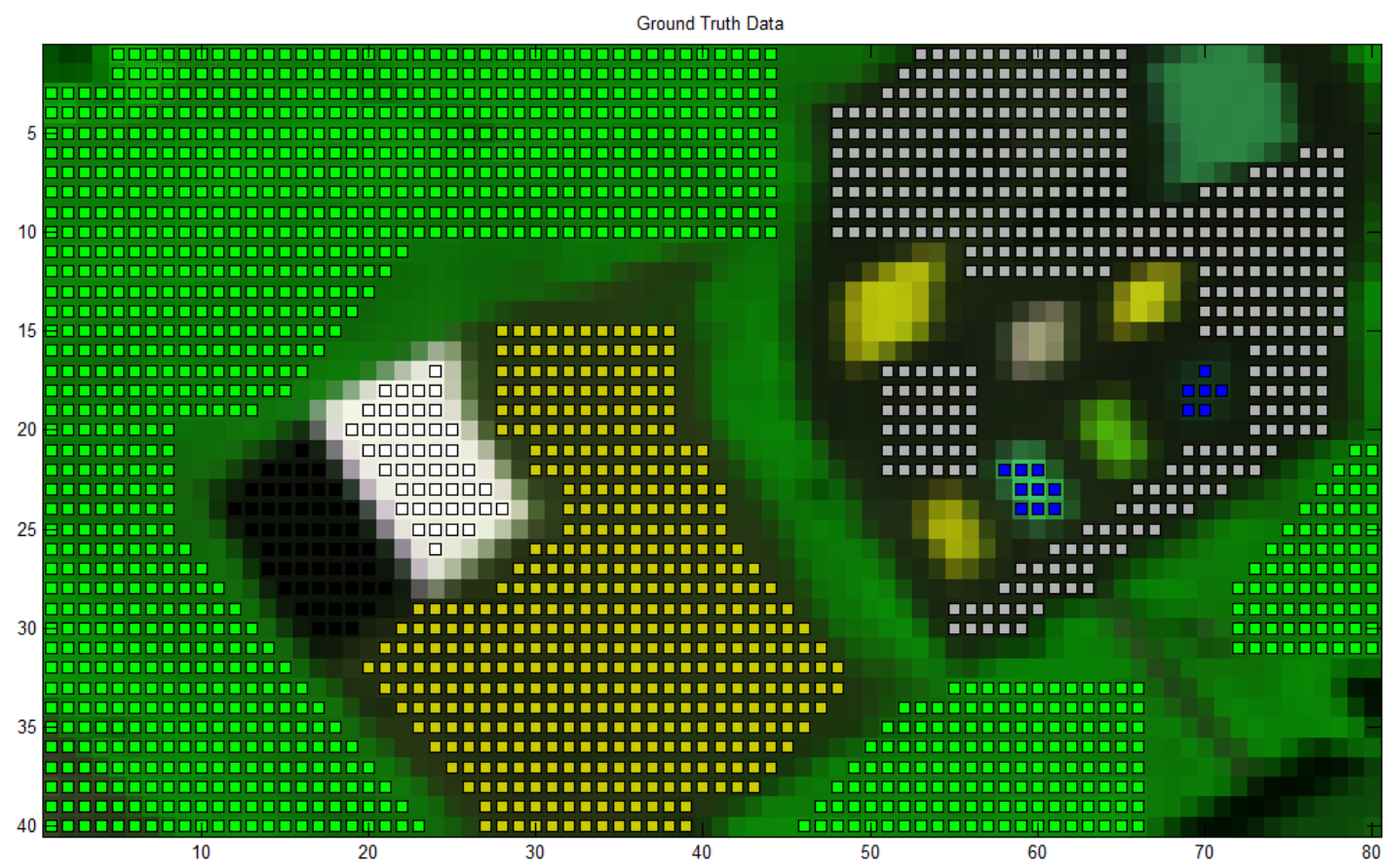

Figure 3: Ground truth pixel image from the HSI data.

\subsection{Target Detection Comparison}

The results for the complete HSI subset containing $80 \times 40$ pixels and 360 bands and give an idea of what to expect from a successful band selection method. Most noticeably, only about $17 \%$ of pixels out of a total 1267 possible are correctly identified. The visual output of classification results is given in figure 4, with quantitative results to follow. Another improvement which has not been quantized, is the amount of misclassifications and large areas that this affects. For example, the majority of grass pixels are classified as gray tarp, which is not present at all in the scene. This shows clear room for improvement.

Now one moves on to the automated band selection results, and the benefits are almost immediately apparent. With a relatively small amount of 15 selected bands, the target detection OA results improve to $32 \%$ for lambda values varying from 0.20 to 0.25 . The output image is given in figure 5 to give a reference between the original results and band selection results without creating a graph with each material classification result. If the eigenvalue threshold lambda is set to a value of 0.20 , there are more bands which can be selected in the maximum distance algorithm. This seems to improve the classification of background targets greatly; however, it is a bit troubling that the blue tarp is no longer classified at all which can be noted by the change between pixel 61,24 in figures 4 and 5 . When lambda is increased to 0.25 , there is a noticeable increase in the number of black tarp pixels which are classified, and the overall accuracy actually increases for these smaller targets.

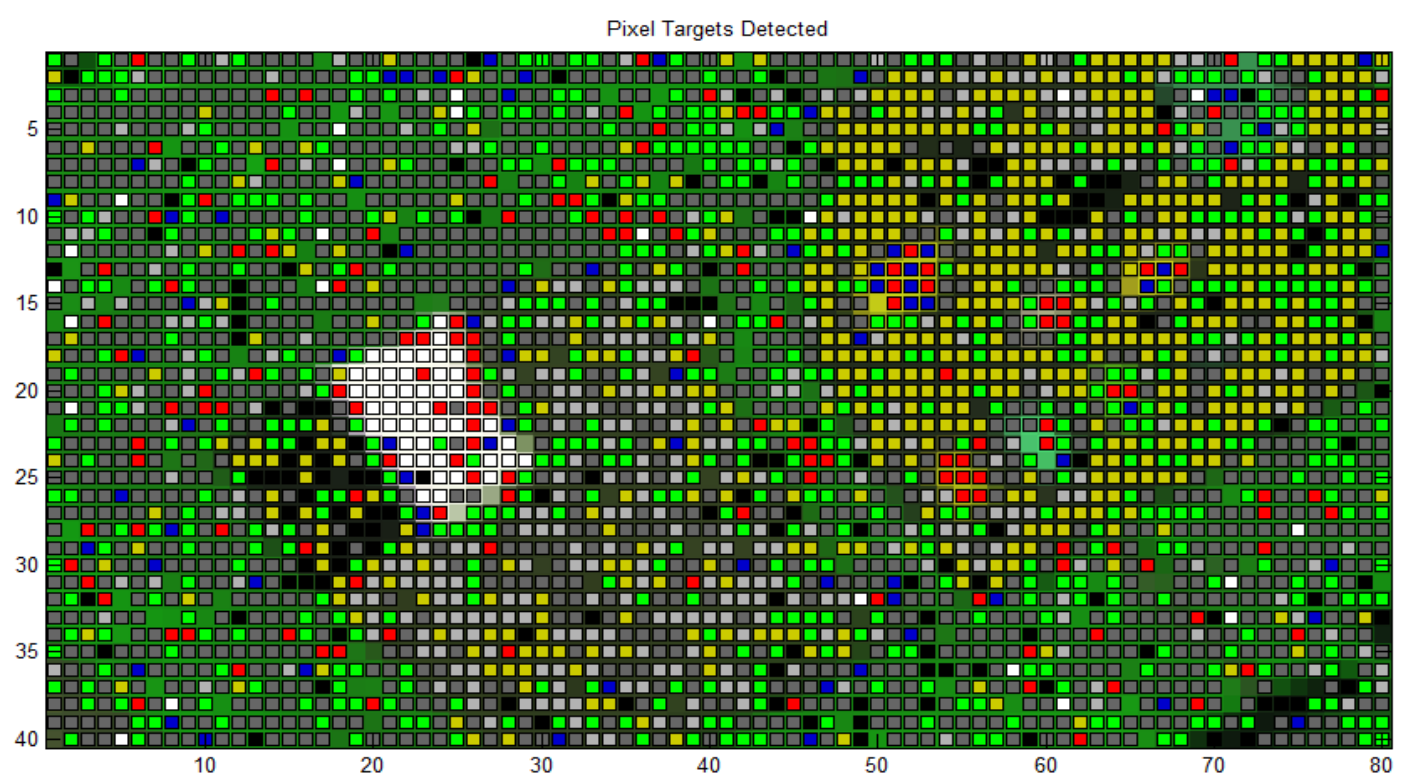

Figure 4: Result for original 360 HSI image bands compared against 12 library spectra. 


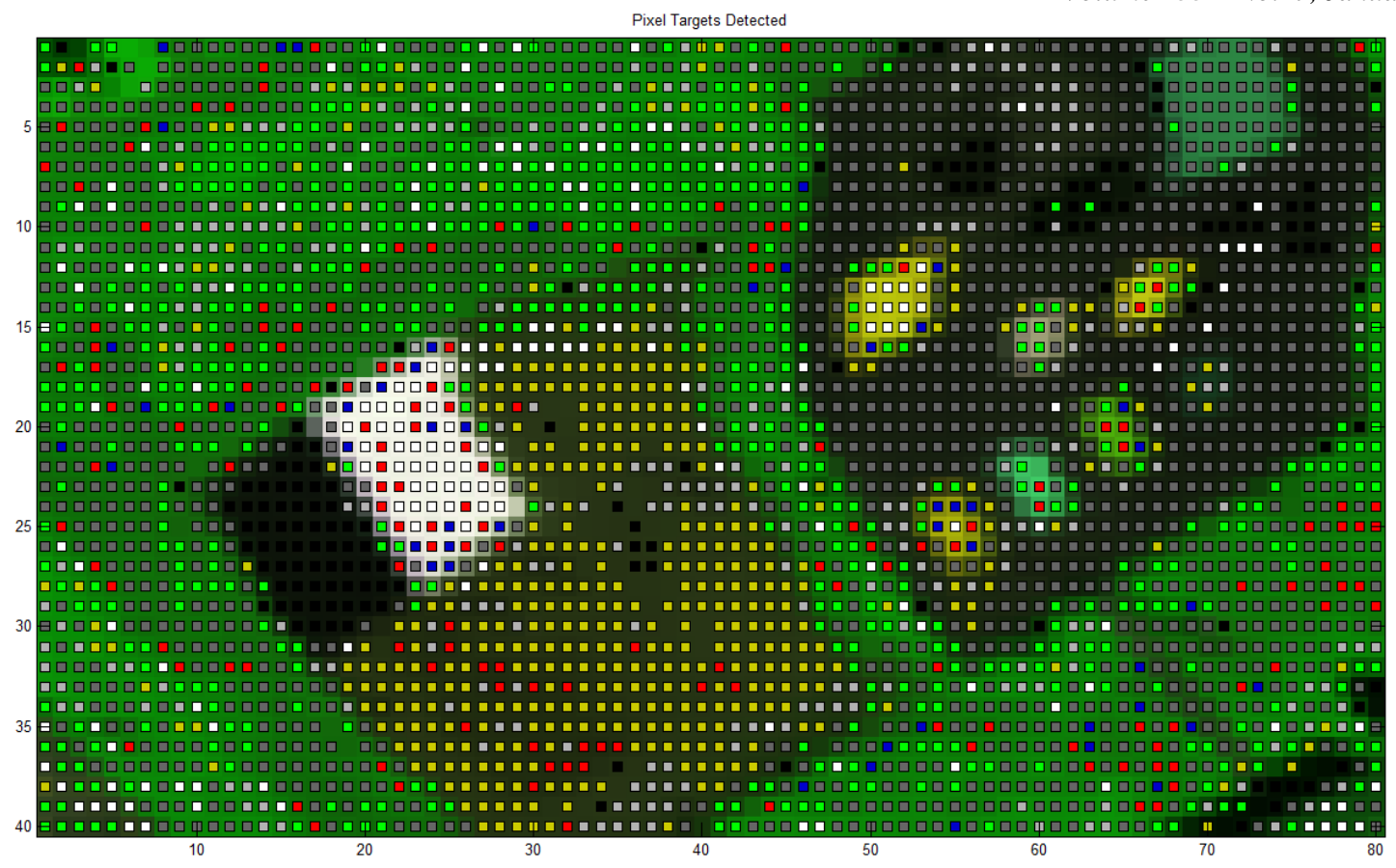

Figure 5: ABS classification results using 15 bands and lambda $=\mathbf{0 . 2 0}$.

In figure 6, a graph is provided which gives the total amount of correctly identified target pixels with respect to the number of bands selected. The amount of bands increments by one, while the lambda is fixed at 0.25 . Then, in figure 7 , the lambda is varied by an increment of 0.01 , and the selection bands are fixed at 20 . The original data only provided $18 \%$ positive identifications, and this is the black hashed line in both figures. Both figures for OA loosely resemble a bathtub curve. Note that if lambda increases above 0.4 less than 20 bands are available for selection due to the high noise content of bands in the dataset. It is clear that more work needs to be done either to optimally select a narrow range of lambda values by performing a case study on several other datasets, or automate the selection of the eigenvalue threshold lambda. To analyze the AA of the results, it is noted that the amount of true negatives is very low due to high amounts of noise in the dataset. Since the noise causes random increase or decrease of reflection values, the SMF target detection algorithm was made to be less sensitive to these changes. This means that a lot of the time, materials are either true or false positives, and there are very few negatives as shown in figure 5 above. The amount of true and false negatives combined only accounts for about $2 \%$ of the total subscene. Therefore, the AA results are weighted heavily by the true positives that are obtained, making this a poor indicator of false alarm rate, and other positive results which occur when the percent of true negatives is very high.

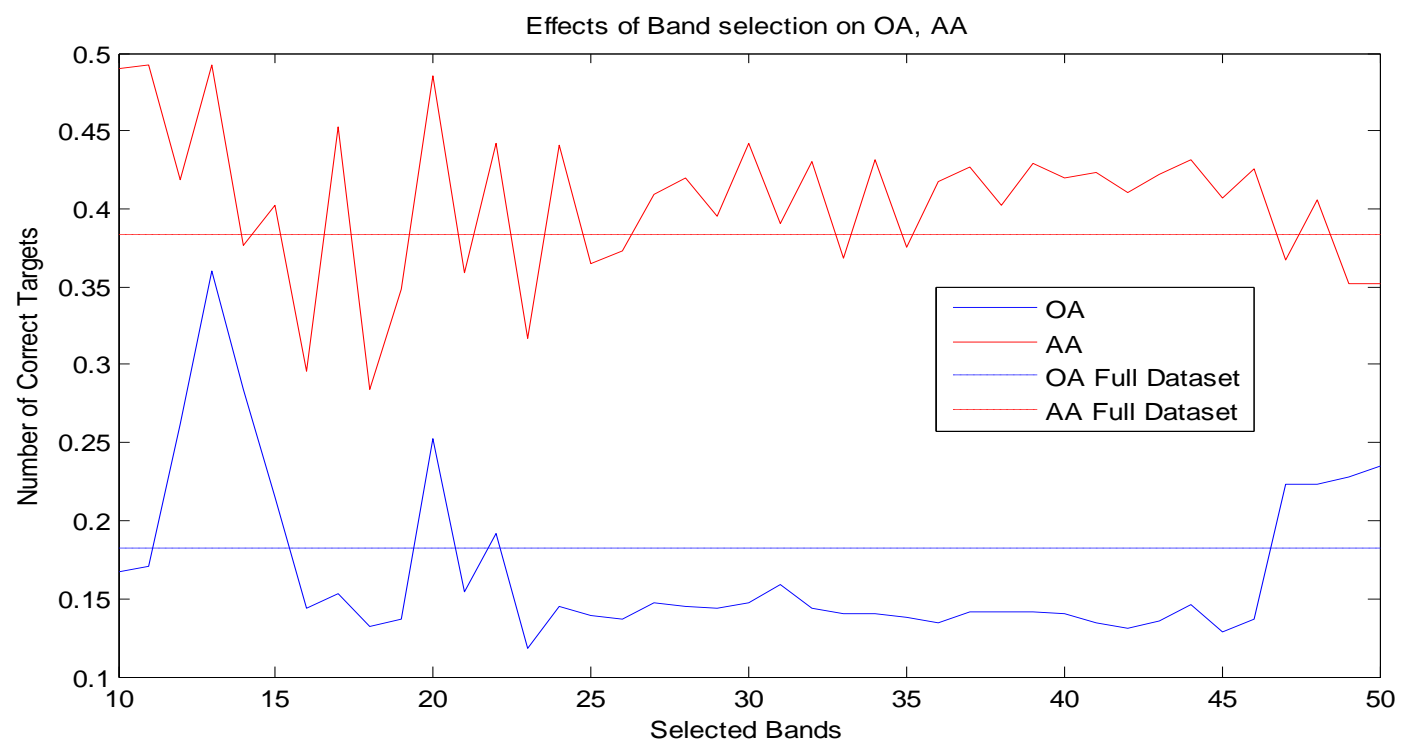

Figure 6: ABS classification results using lambda $=\mathbf{0 . 2 5}$, varied number of selected bands. 


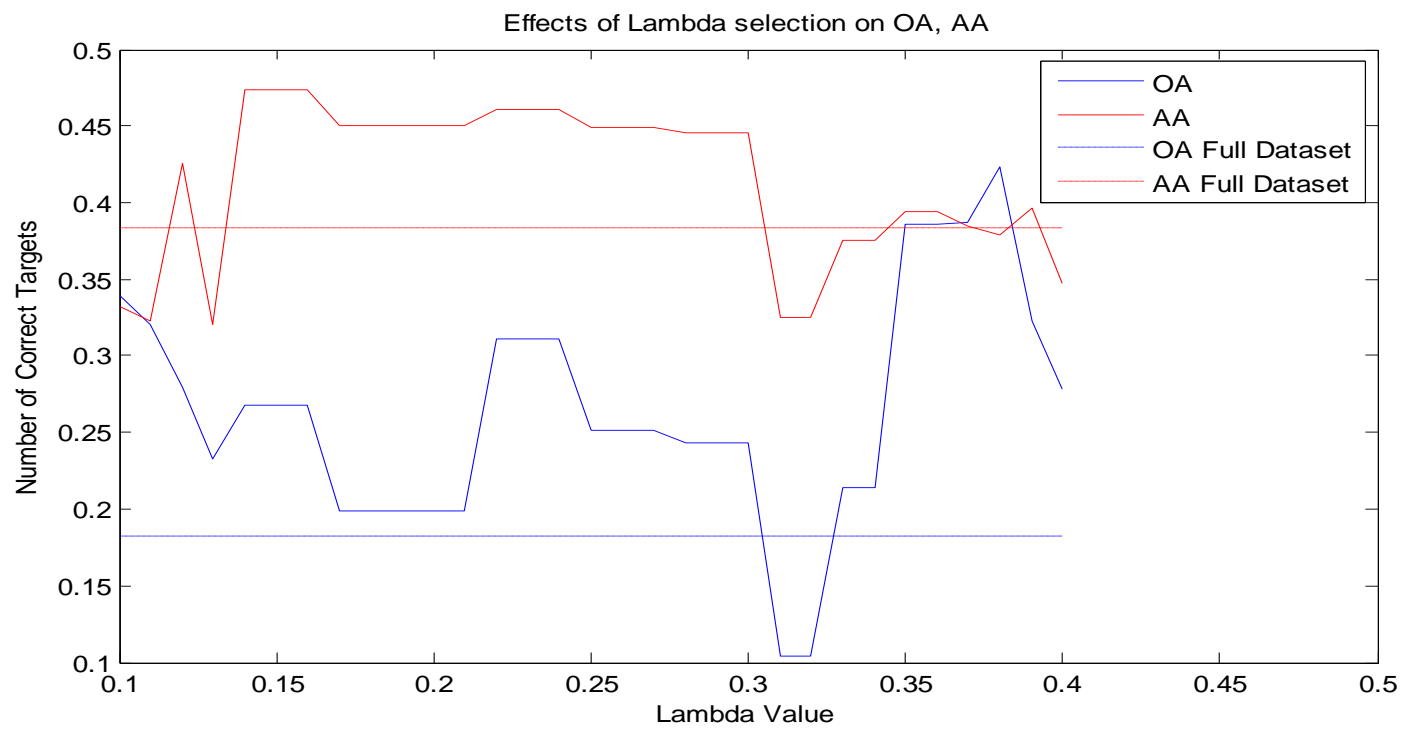

Figure 7: ABS classification results using 20 bands, varied lambda values.

\subsection{Run-time Comparison Results}

Intuitively, it is expected that the run time to be inversely proportional to lambda, since a lower lambda value causes the maximum distance algorithm to sort through a larger amount of bands, and those bands have higher levels of noise which lead to difficult classification. It follows that with an increased number of selected bands, that the SMF target detection algorithm will become more complex: requiring more time as well. It is also interesting to note that the maximum distance algorithm will have to compare more bands in the cluster to the incoming band; however this proves less costly than the target detection itself. Using 15 bands and values of lambda as outlined in the target detection results, CPU time is reduced throughout the ABS runtime data.

The results for comparing runtime against the number of selected bands is given in figure 8 , and it is immediately clear that lambda has limited effect on the time required for target detection. This is because the only thing that happens when lambda is decreased, is that more eigenvalues are compared against the cluster in the maximum distance algorithm. This is far less time consuming than the comparison of the pixels to the library spectra, which is reduced by the number of bands required for comparison. The effect of increasing the number of bands used in the SMF is shown in figure 9.

With only 15 ABS bands, and a lambda of 0.25 , the target detection algorithm takes only 5.96 seconds to complete, compared with 32.4 seconds using the full 360 band dataset. This is a substantial decrease showing 5 times speedup, showing the greatest amount of potential for this method. This is nearly comparable to the results shown in the Fast Selection method proposed by $\mathrm{Du}$ [14], in which the speedup is 6.6 with a larger dataset using GPU implementation. There are no classification results because the goal is band selection and not the repercussions of the method on target detection. Another paper regarding the N-Findr band selection method by Wang [15], a 20 times speedup is discussed between existing methods and the new method, but this is only the time required for band selection, and gives no classification results.

As the eigenvalues are calculated for every single band in the HSI dataset, there is an asymptote to the amount of speedup that can be achieve using this method. For the RIT dataset, it takes 2.83 seconds to calculate all of the eigenvalues for 360 bands. This means that at $15 \mathrm{ABS}$ bands, this process begins to dominate the SMF comparison algorithm requiring $47 \%$ of the total 5.96 seconds in order to determine the quality of each band. This algorithm could be parallelized in future works to avoid bottlenecking.

The time reduction benefits should become more apparent when using a larger image, since target detection time begins to take the majority of computational time, and outweighs the amount of time required for band selection. This is due to the fact that the same number of eigenvalue calculations are performed as in this test image, although they become more complex. Further investigation on the effects of calculating these band quality values is necessary to validate this theory, and the possibility of local grouping to decrease runtime further. 


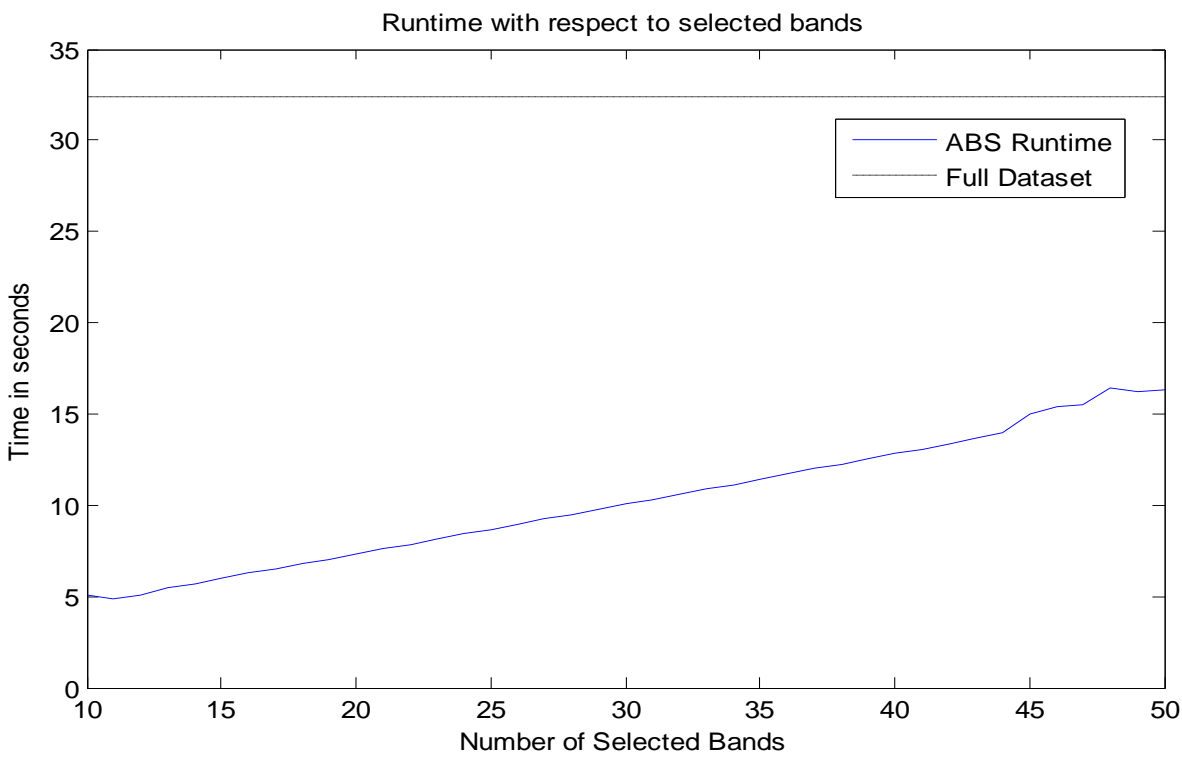

Figure 8: Target detection runtime results using 10 to 50 bands and lambda $=0.25$.

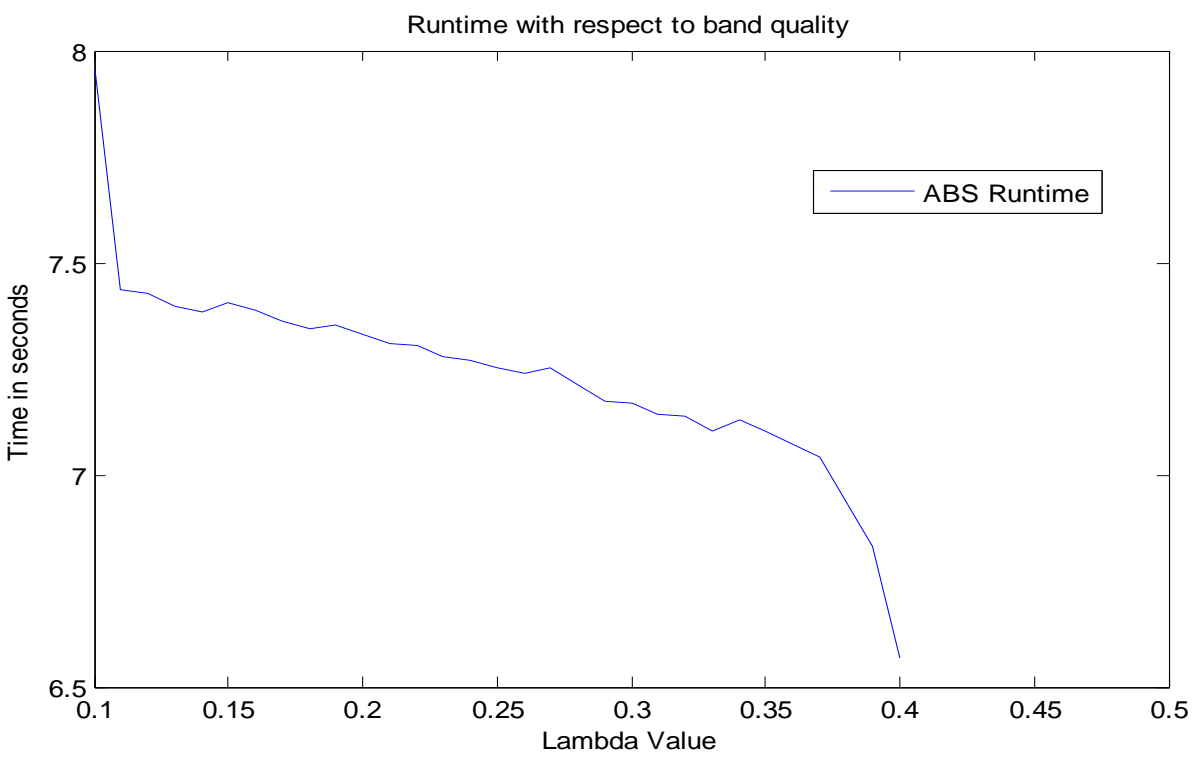

Figure 9: Target detection runtime results varying lambda with 20.

\section{FUTURE WORK AND CONCLUSION}

It is interesting to note, that as the value of lambda increases greatly, near the maximum possible value of 0.4 , the background classes such as grass and sand are classified very well, but targets in the scene such as the white tarp and gravel areas are almost entirely misclassified. This is because the selection process is beginning to eliminate bands which contain information. On the other hand, when lambda is decreased until nearly all of the bands are present after preprocessing, the maximum distance algorithm seems to favor noise bands due to their high covariance between other bands. This leads to errors caused by atmospheric scattering and other sources of noise.

One should look into the effects of this band reduction method on other datasets such as Indian Pines, to make it favorable for comparison with other methods that use this well known dataset. In addition, larger block sizes should be used to investigate the effects of scaling this algorithm.

It is reasonable to think that one could use multispectral imaging based on the result that 15 bands is enough to correctly classify an image; however, these bands would have to be carefully selected prior to airborne data collection which is simply impossible based on the variation between scenes, and atmospheric effects which may vary due to temperature or time of day.

Although the initial goal was to find a preprocessing method to reduce the time required for target detection, this automated band selection method improves the overall accuracy of target detection more than the computational time. Further research is necessary to simplify calculations within the band reduction preprocessing algorithm to reduce computation time even further. Finally, the classification results suggest that the band 
selection method produces groups with similar classification results within the same area. This means that in addition to increasing overall accuracy, the algorithm actually makes the results of target detection more predictable, and less random based on the presence of noise bands. This may lead to further research in nearest neighbor and local target prediction algorithms.

\section{ACKNOWLEDGEMENTS}

This work was supported in part by the Nevada EPSCoR program, NSF award \#EPS-IIA-1301726, and by the DODDTRA Grant/Award \#: HDTRA1-12-1-0033. Additional guidance and library data was provided in collaboration with Todd Morken and RSL.

\section{REFERENCES}

[1] Purdue Research Foundation, "Aviris image Indian Pine Test Site," 2014, https://engineering.purdue.edu/ biehl/MultiSpec/hypersp ectral.html.

[2] Caltech, "AVIRIS Overview," 2000, http://aviris.jpl.nasa.gov/html/overview.html.

[3] SpecTIR Remote Sensing Division, "ProSpecTIR VS," 2011, http://www.spectir.com/wpcontent/uploads/2012/02/ProSpecTIR_VS_specs_2011.p df.

[4] A. Giannandrea, N. Raqueno, D. Messinger, J. Faulring, J. Kerekes, J. van Aardt, K. Canham, S. Hagstrom, E. Ontiveros, A. Gerace, J. Kaufman, K. Vongsy, H. Griffith, and B. Bartlett, "The SHARE 2012 data collection campaign," 2013. <http://dx.doi.org/10.1117/12.2015935>

[5] D. Manolakis, R. Lockwood, T. Cooley, J. Jacobson, "Robust Matched Filters for Target Detection in Hyperspectral Imaging Data," Acoustics, Speech and Signal Processing, 2007. ICASSP 2007. IEEE International Conference on. Vol. 1. IEEE, 2006. <http://dx.doi.org/10.1109/IGARSS.2006.99>

[6] O. Rajadell, P. Garcia-Sevilla, V. C. Dinh, and R. P. W. Duin, "Improving Hyperspectral Pixel Classification With Unsupervised Training Data Selection," 2014. <http://dx.doi.org/10.1109/LGRS.2013.2273983>
International Journal of Computer Applications (0975 - 8887)

Volume 133 - No.17, January 2016

[7] K. Sun, X. Geng, L. Ji, and Y. Lu, "A New Band Selection Method for Hyperspectral Image Based on Data Quality," 2014 <http://dx.doi.org/10.1109/JSTARS.2014.2320299>

[8] Q. Du, and H. Yang, "Similarity-based unsupervised band selection for hyperspectral image analysis," Geoscience and Remote Sensing Letters, IEEE5.4, 564-568, 2008, <http://dx.doi.org/10.1109/LGRS.2008.2000619>

[9] T. Lin, and S. Bourennane. "Hyperspectral image processing by jointly filtering wavelet component tensor," Geoscience and Remote Sensing, IEEE Transactions on 51.6, 3529-3541, 2013, <http://dx.doi.org/10.1109/TGRS.2012.2225065>

[10] Y. Qian, F. Yao, and S. Jia, "Band selection for hyperspectral imagery using affinity propagation," IET Comput. Vis., vol. 3, no. 4, pp. 213-222, 2009, <http://dx.doi.org/10.1049/iet-cvi.2009.0034>

[11] M. Griffin, and H. H. Burke, "Compensation of hyperspectral data for atmospheric effects," Lincoln Laboratory Journal, 14(1), 29-54, 2003 <http://dx.doi.org/10.1.1.73.727>

[12] Rochester Institute of Technology, Flexviewer, http://barracuda.cis.rit.edu/flexviewer/.

[13] K. N. Liou, An introduction to atmospheric radiation. vol. 84. Academic press, 2002. page 86

[14] H. Yang, and Q. Du, "Fast band selection for hyperspectral imagery," 2011 IEEE 17th International Conference on Parallel and Distributed Systems, 2011. <http://dx.doi.org/10.1109/ICPADS.2011.157>

[15] L. Wang, Y. Zhang, and Y. Gu, " Unsupervised Band Selection Method Based on Improved N-FINDR Algorithm for Spectral Unmixing," ISSCAA Preceedings pp.10211024,2006.http://dx.doi.org/10.1109/ISSCAA.20 06.1627496

[16] BS ISO 5725-1: "Accuracy (trueness and precision) of measurement methods and results - Part 1: General principles and definitions.", p.1 (1994) 